\title{
GROUPS OF ODD ORDER IN WHICH EVERY SUBNORMAL SUBGROUP HAS DEFECT AT MOST TWO
}

\author{
JOHN COSSEY \\ (Received 23 November 1989; revised 24 October 1990)
}

Communicated by H. Lausch

\begin{abstract}
In 1980, McCaughan and Stonehewer showed that a finite soluble group in which every subnormal subgroup has defect at most two has derived length at most nine and Fitting length at most five, and gave an example of derived length five and Fitting length four. In 1984 Casolo showed that derived length five and Fitting length four are best possible bounds.

In this paper we show that for groups of odd order the bounds can be improved. A group of odd order with every subnormal subgroup of defect at most two has derived and Fitting length at most three, and these bounds are best possible.
\end{abstract}

1980 Mathematics subject classification (Amer. Math. Soc.) (1985 Revision): 20 D 35, 20 E 34.

\section{Introduction}

Groups in which every subnormal subgroup is normal (so that normality is transitive, and hence the name $T$-groups) have been extensively studied, the study of finite soluble $T$-groups was begun by Zacher [15] in 1952. Gaschütz [3] gave a detailed picture of their structure in 1957, Robinson [12] and Peng [11] provided some additional detail. A summary of their structure can be found in Robinson [13].

In 1980, McCaughan and Stonehewer [10] began the study of finite soluble groups in which every subnormal subgroup has defect at most two (we call this class of groups $\mathscr{B}_{2}$, following McCaughan and Stonehewer). They showed that groups in $\mathscr{B}_{2}$ have bounded derived and Fitting lengths, the bounds they obtained being 9 and 5 respectively. They also observed that the split

(C) 1991 Australian Mathematical Society 0263-6115/91 \$A2.00+0.00 
extension of the natural module for $\operatorname{GL}(2,3)$ by $\operatorname{GL}(2,3)$ is a group in $\mathscr{B}_{2}$ of derived length 5 and Fitting length 4 . Their bounds have been improved to 5 and 4 respectively by Casolo [2], and these bounds are thus best possible.

Our aim in this paper is to show that these bounds can be improved for groups of odd order. We show that the best possible bound for both derived and Fitting length is 3 for odd order groups in $\mathscr{B}_{2}$. In fact we will prove a little more. Any finite group can be written as a subdirect product of monolithic groups (that is, groups with a unique minimal normal subgroup); since $\mathscr{B}_{2}$ is quotient closed, any group in $\mathscr{B}_{2}$ can be written as a subdirect product of monolithic groups in $\mathscr{B}_{2}$ (though not every subdirect product of groups in $\mathscr{B}_{2}$ is in $\mathscr{B}_{2}$ ). Thus to establish bounds for the derived or Fitting length it is enough to consider monolithic odd order groups in $\mathscr{B}_{2}$. We show that the structure of a monolithic odd order group in $\mathscr{B}_{2}$ is quite restricted. Note that for a monolithic group, the Fitting subgroup must be a $p$-group for some prime $p$.

THEOREM. Let $G$ be a monolithic group of odd order in $\mathscr{B}_{2}$ and let $p$ be the prime dividing the order of $F(G)$. Then one of the following holds.

(i) $G$ is nilpotent of class at most 3.

(ii) $F(G)$ is nonabelian and $G / F(G)$ is an abelian $p^{\prime}$-group.

(iii) $F(G)$ is abelian and complemented in $G$, by $H$ say. Moreover, $O_{q}(H)$ is cyclic if $q$ does not divide $p-1$; and if $q$ divides $p-1$, either $O_{q}(H)$ is abelian and every chief factor $K / L$ of $H$ with $K \leq$ $O_{q}(H)$ has $|K / L| \leq q^{2}$ or $O_{q}(H)$ is nonabelian and every chief factor $K / L$ of $H$ with $K \leq O_{q}(H)$ is central.

That a monolithic odd order group in $\mathscr{B}_{2}$ has derived and Fitting length at most 3 is an easy corollary. If $F(G)$ is nonabelian then since $F(G)$ is in $\mathscr{B}_{2}$ we have $F(G)$ metabelian by (i) and so $G$ clearly has derived length at most 3 and Fitting length at most 2. If $F(G)$ is abelian let $H$ be a complement for $F(G)$ in $G$. It follows immediately from (iii) that if $K / L$ is a chief factor of $H$ with $K \leq F(H),|K / L| \leq q^{2}$, for some prime $q$. Thus $H / C_{H}(K / L)$ is either cyclic or an odd order subgroup of $\mathrm{GL}_{2}(q)$, and it then follows that $H / C_{H}(K / L)$ is abelian (using Curtis and Reiner [1, Theorem 53.17] for example). Since $F(H)$ is the intersection of the centralisers of chief factors of $H$ contained in $F(H)$, we get $H / F(H)$ is abelian. If $q$ is a prime dividing $|F(H)|$, we let $C$ be a normal subgroup of $H$ chosen maximal such that $C \cap O_{q}(H)=1$. We then have that $F(H) \leq C . O_{q}(H)$ and so $H / C \cdot O_{q}(H)$ is abelian. Thus if $O_{q}(H)$ is abelian $H / C$ is metabelian. If $O_{q}(H)$ is nonabelian, we have all $q$ chief factors of $H / C$ central and hence 
$H / C$ is a $q$-group and since it is also in $\mathscr{B}_{2}, H / C$ is again metabelian. Finally $H$ is metabelian, being a subdirect product of metabelian groups. To see that this bound is best possible, let $p, q$ be odd primes with $q$ dividing $p-1$, let $H$ be a nonabelian group of order $p q$, and let $U$ be a faithful irreducible $G F(q) H$-module. Then $G=U H$ is a group in $\mathscr{B}_{2}$ of derived and Fitting length 3.

\section{Preliminaries}

Our notation is mostly standard. The following should be noted. We denote the centre of $G$ by $\zeta(G)$, the $i$ th term of the lower central series of $G$ by $\gamma_{i}(G)$, and the socle of $G$ by $\sigma(G)$. The class of $q$-nilpotent soluble groups, $q$ a prime, is denoted by $\mathscr{S}_{q^{\prime}} \mathscr{S}_{q}$, and the class of finite soluble groups of nilpotent length at most $n$ is denoted by $\mathscr{N}^{n}$. If $\mathscr{F}$ is a class of finite soluble groups closed under taking subdirect products, and $G$ is a finite group, we call the smallest normal subgroup $N$ such that $G / N$ is in $\mathscr{F}$ the $\mathscr{F}$-residual of $G$ and denote it by $G^{\mathscr{F}}$.

For the remainder of this paper, all groups are finite and of odd order. Throughout this section, let $p, q$ be distinct odd primes and let $K$ be a field of characteristic $p$.

There are a number of results we will need to use frequently, most are well known. Results concerning modules for which no reference is given can be found in Huppert [7] or Curtis and Reiner [1]. We will also need the following results.

LEMMA 1. Let $A$ be an abelian group on which $H$ acts as a group of operators. Let $N$ be a normal subgroup of $H$ of order coprime to $|A|$. Then $A=$ $B \times C$, where $B$ and $C$ are $H$-subgroups of $A$ and $[B, N]=1,[C, N]=C$.

For a proof see Higman [6].

LEMMA 2. Let $G$ be a group with a faithful irreducible representation over $K$. If $N$ is a normal subgroup of $G$ with $N \leq \sigma(G)$ then $N$ is the normal closure of a single element.

This is a special case of a theorem of Gaschütz [4].

LEMma 3. Let $G$ be a p-nilpotent group and $U$ an indecomposable $K G$ module. Then all the composition factors of $U$ are isomorphic.

For a proof see Huppert and Blackburn [8, Theorem VII.14.9]. 
The next group of lemmas are technical ones we will need in the proof of the main theorem.

LemMA 4. Let $H=Q R$, where $Q$ is a nonabelian normal $q$-subgroup and $R$ is a $q^{\prime}$-group. Suppose that $Q=M N$, where $M, N$ are abelian subgroups of $Q$ with $M, N$ normal in $H$ and $Q / M, Q / N$ noncentral cyclic chief factors of $H$. If $Q / M, Q / N$ are nonisomorphic as $H$-modules then $H$ is not in $\mathscr{B}_{2}$.

Proof. Note that if we set $L=M \cap N, L \leq \zeta(Q)$. Since quotients of groups in $\mathscr{B}_{2}$ are also in $\mathscr{B}_{2}$, it will be enough to prove the lemma for the case where $Q^{\prime}$ is a minimal normal subgroup of $H$. Moreover, $L$ must be cyclic (so that all chief factors of $H$ in $L$ are isomorphic as $H$-modules). Note that $R$ acts on $Q / M$ and $Q / N$ as distinct power automorphisms and hence on $Q^{\prime}$ as a power automorphism distinct from both of these. Thus $M, N$ cannot be cyclic and it is now easy to see that $L=Q^{\prime}$ and $Q$ has order $q^{3}$ and exponent $q$. But then we can choose an element $x$ with $x$ in neither $M$ nor $N$ such that $\langle x\rangle^{H}=Q$. Since $\langle x\rangle$ is not normal in $Q$ we have $H \notin \mathscr{B}_{2}$.

LeMma 5. Let $H$ be a group with an elementary abelian normal $q$-subgroup $N$ such that $N$ is the normal closure of a single element, $|N| \geq q^{3}$ and either $N$ has at most one subgroup of index $q$ which is normal in $H$ or $H / C_{H}(N)$ is cyclic. Let $U$ be a faithful irreducible $K H$-module, let $W$ be a homogeneous component of $U_{N}$ and set $C=C_{N}(W)$. Then for some $h$ in $H$ we have $W^{h} \neq W, C^{h} \neq C$ but $\left(C^{h} \cap C\right)^{H}=N$. If $X$ is a diagonal subspace of $W \oplus W^{h}$ and $V=X \oplus Y$, where $Y$ is a complement for $W \oplus W^{h}$ in $U_{N}$, then $U / V$ is trivial as a $\left(C^{h} \cap C\right)$-module but $V$ is not an $N$-submodule of $U$.

Proof. Since $\bigcap_{h \in H} C^{h}=1$ we have $C \neq C^{h}$ for some $h$ in $H$ and then $W \neq W^{h}$. Set $C \cap C^{h}=C_{h}$ and suppose that $C_{h}^{H} \neq N$ for any $h$ such that $C_{h} \neq C$.

Suppose first that $N$ has a unique maximal subgroup $M$ with $M$ normal in $H$. Then we must have $C_{h}^{H} \leq M$. Since $|N / C|=q,\left|N / C_{h}\right|=q^{2}$. Moreover $C \cap M$ cannot be $M$ and so we get $M>C \cap M \geq C \cap M \cap C^{h}=$ $C_{h}$. Thus $C \cap M=C_{h}$ and $M>(C \cap M)^{h}=C^{h} \cap M \geq C_{h}$, giving $(C \cap M)^{h}=C \cap M$ for all $h$ in $H$. But $C \cap M \neq 1$ and $C$ can contain no nontrivial normal subgroup of $H$, a contradiction. 
Hence suppose that $H / C_{H}(N)$ is cyclic. If $h$ is an element of $H$ such that $h C_{H}(N)$ generates $H / C_{H}(N)$, then $C_{h}^{H}=M \neq N$. As above we see that $C \cap M=C^{h} \cap M$ and hence $(C \cap M)^{a}=C \cap M$ for all $a$ in $H$, again giving a contradiction.

To complete the proof of the lemma, observe that $X=V \cap\left(W \oplus W^{h}\right)$ would be an $N$-submodule if $V$ were, and $X$ is clearly not an $N$-submodule.

LEMMA 6. Let $H$ be a group in $\mathscr{B}_{2}$ and suppose $H=Q R$, where $Q$ is a q-group and is normal in $H$ and $R$ is a p-nilpotent group whose order is prime to $q$. Suppose that $Q$ is nonabelian and $[Q, R] \neq 1$. Suppose further that $U$ is a faithful irreducible $K H$-module. Then there is a subgroup $S$ of $Q$ and a subspace $V$ of $U$ such that $V$ is an $S$-submodule of $U$ and $S$ acts trivially on $U / V$ but $V$ is not an $S^{H}$-submodule of $U$.

Proof. We suppose the lemma is not true and let $H, U$ be a counterexample with $H$ chosen to have smallest possible order.

If $M$ is a maximal normal subgroup of $H$ with $Q \leq M$, then $|H / M|$ is prime to $q$. We have $U_{M}$ completely reducible so let $U_{M}=W \oplus X$, with $W$ irreducible and set $C=C_{H}(W)$. Since $O_{q}(M / C)=Q C / C$ and $\bigcap_{h \in H} C^{h}=$ 1 we have $O_{q}(M / C)$ nonabelian. It then follows from the minimality of $H$ that if $T$ is a Hall $q^{\prime}$-subgroup of $M$ and $\left[O_{q}(M / C), T C / C\right] \neq 1$ then there is a subgroup $S / C \leq O_{q}(M / C)$ and an $S / C$ submodule $V$ of $W$ with $S / C$ (and hence $S$ ) acting trivially on $W / V$ and with $V$ not an $(S / C)^{M / C}$ submodule of $W$. It is then clear that if $S_{q}$ is a Sylow $q$-subgroup of $S$ then $V \oplus V$ is an $S_{q}$-submodule of $U$ with $S_{q}$ acting trivially on $U /(V \oplus X)$, but $V \oplus X$ is not an $S_{q}^{H}$-submodule of $U$. Thus $H, U$ is not a counterexample, a contradiction. Hence we may assume that $[Q C / C, T C / C]=1$ and since this holds for each irreducible constituent of $U_{M}$ we may assume that $[Q, T]=1$.

Next choose $M$ maximal such that $M$ is normal in $H$ and properly contained in $Q$. Consider $U_{M R}$. Since $H$ is $p$-nilpotent, by Lemma $3 U_{M R}$ is a direct sum of indecomposable modules each of which has all its composition factors isomorphic. Let $W$ be an irreducible submodule of $U_{M R}$ and $C=C_{M R}(W)$. Moreover, $U_{M}$ is a direct sum of conjugate irreducible modules and as before, if $M$ is nonabelian then $M C / C$ is nonabelian. If as well $[M C / C, R C / C] \neq 1$ we again get a contradiction. Thus either $M$ is abelian or $[M, R]=1$.

Suppose that $M=\Phi(Q)$. Then $Q / M$ is an irreducible module for the group $R / T$ of prime order. Since $Q$ is not cyclic, any noncentral $q$-chief factor of $H$ must have order at least $q^{2}$. We consider the cases $[M, R]=1$ 
and $[M, R] \neq$ separately. If $[M, R]=1$ then $M=Q^{\prime}$ since $Q / Q^{\prime}$ can contain no elements centralised by $R$ (by Lemma 1). Moreover, if $Q$ has class greater than 2 we would have $\gamma_{3}(Q) / \gamma_{4}(Q)$ is clearly not centralised by $R$ if $\gamma_{2}(Q) / \gamma_{3}(Q)$ is; hence $Q$ must have class 2 and $M$ is abelian. Since $Q / Q^{\prime}$ has exponent $q$, so does $Q^{\prime}$ and since $Q^{\prime}$ is central in $H$ it must be cyclic by Lemma 2. Thus $|M|=q$ and $Q$ is itself monolithic. By Huppert [7, Hilfssatz III.12.2], $Q$ contains an element $x$ of order $q$ not in $M$. If $U_{Q}=W \oplus X$ with $W$ irreducible, $W$ is clearly faithful. Thus $W_{\langle x\rangle}$ is faithful and so $0 \neq[W, x]=\{w x-w: w \in W\} \neq W$. Put $S=\langle x\rangle$ and $V=[W, x] \oplus X$. Then $V$ is an $S$-submodule of $U, S$ acts trivially on $U / V$ and $S^{H}=Q$, so that $V$ is not an $S^{H}$-submodule of $U$. If $[M, R] \neq 1$ then $M$ is abelian and it follows that $\sigma(M)$ contains a subgroup $N$ which is the normal closure of a single element and contains a nontrivial chief factor of $H$. If $|Q / M| \geq q^{3}$ then $|N| \geq q^{3}$ and Lemma 5 gives a contradiction. Thus $|Q / M|=q^{2}$. We then have that $\left[R, Q^{\prime}\right] \leq \gamma_{3}(Q)$ and hence $\sigma(M)$ contains both central and noncentral chief factors of $H$, giving that $\sigma(M)$ contains a subgroup $N$ which is the normal closure of a single element in $H$ and with $|N| \geq q^{3}$. Again Lemma 5 gives a contradiction and so it follows that $M$ cannot be abelian.

If $Q / M$ is a central chief factor of $H$, we must have $[M, R] \neq 1$ and hence abelian. Again by Lemma 1 we have $M=A \times B$, where $A, B$ are normal in $H$ and $[A, R]=1,[B, R]=B$; note that $B \neq 1$. We also have that $B$ is the $\mathscr{S}_{q}, \mathscr{S}_{q}$ residual of $H$ and so is complemented in $H$ (Huppert [7, VI.7.15]). If $S$ is a Sylow $q$-subgroup of a complement then $S$ is a complement for $B$ in $Q$. Suppose that $[S, B]=1$. Then we must have $Q=S \times B, H=S \times(B R)$, and $S$ nonabelian. By Huppert [7, Satz III.12.3], $S$ contains an elementary abelian normal subgroup $E$ of order $q^{2}$. Since $H$ is faithfully and irreducibly represented on $U$, and $E$ is normal in $H$, it must be the normal closure of a single element. If $D \leq \sigma(B)$ is a minimal normal subgroup of $H$ then $N=E \times D$ satisfies the hypotheses of Lemma 5 and which then gives a contradiction. Thus for some $x$ in $S$ we have $[B, x] \neq 1$ and the minimality of $H$ then gives $S=\langle x\rangle$. If $U_{Q}=W_{1} \oplus \cdots \oplus W_{t}$, where the $W_{i}$ are the homogeneous components of $U_{Q}$, we have $t>1$ and we may suppose that $W_{1 D}$ and $W_{2 D}$ are nonisomorphic, where $D \leq \sigma(B)$ is minimal normal and noncentral in $H$. Noting that $Z=\left\langle x^{q}\right\rangle$ is central in $H$, we have $U_{Z}$ is homogeneous. If $X$ is an irreducible submodule of $W_{1}$ then we have $X=Y^{Q}$, where $Y$ is an irreducible $K M$-module. It then follows from the Mackey subgroup theorem that $X_{S}=Y_{Z}^{S}$ and hence the $W_{i S}$ are all isomorphic. Let $V_{0}$ be a diagonal $K S$-submodule of $W_{1} \oplus W_{2}$ and set $V=V_{0} \oplus W_{3} \oplus \cdots \oplus W_{t}$. Then $S, V$ 
satisfy the hypotheses and conclusion of the lemma, giving a contradiction.

Thus we may assume that there is no $M$ with $Q / M$ a central chief factor. If

$$
Q / \Phi(Q)=\left(M_{1} / \Phi(Q)\right) \times \cdots \times\left(M_{t} / \Phi(Q)\right)
$$

with $M_{i} / \Phi(Q)$ a (noncentral) chief factor of $H$, then each $M_{i}$ is abelian and so $\left[M_{i}, M_{j}\right] \neq 1$ for some $i, j$. If $t>2$ then $M_{i} M_{j} \leq M$ for some $M$ maximal in $Q$ with $M$ normal in $H$. But then $M$ is abelian, a contradiction. Hence $Q=M_{1} M_{2}$ and since $M_{1}, M_{2}$ are both abelian, $Q$ has class 2 with $\Phi(Q) \leq \zeta(Q)$. Set $L=\Phi(Q)=M_{1} \cap M_{2}$ and suppose that $M_{1} / L$ is noncyclic. Since $Q$ can contain no elementary abelian subgroup normal in $H$ of order greater than $q^{3}, M_{i}$ must be homocyclic and indecomposable as $R$-group and $\left|M_{i} / L\right|=q^{2}, i=1,2$ (this follows easily from Taunt [14, Lemma 6.1 and Corollary 6.2]). We thus have that $L$ is the unique maximal subgroup of $M_{i}$ normal in $H$ and $M_{i} / L$ is isomorphic as $R$-module to $\sigma(L)$. It is easy to see that if $M$ is any subgroup of $Q$ such that $Q / M$ is a chief factor of $H$ then $M$ is homocyclic. If $x$ is an element of $Q$ not in $L$ and $M$ is the normal closure of $\langle x\rangle$ then we have $Q / M$ a chief factor of $H$ and hence $x$ does not have order $q$. But then, since $Q$ cannot be generated by 3 elements it has an abelian normal subgroup (and hence an elementary abelian normal subgroup, $S$ say) requiring at least 3 generators, by Huppert [7, Satz III.12.3]. Since we must have $S \leq L$ and $L$ is a 2-generator group we have a contradiction.

Thus we can assume $M_{1} / L$ and $M_{2} / L$ cyclic and hence $Q$ is a 2 generator group of class 2. By Lemma 4 we can assume that $M_{1} / L$ and $M_{2} / L$ are isomorphic as $H$-modules and hence $Q^{\prime}$ is not central in $H$. Note that any subgroup of $Q$ containing $Q^{\prime}$ is then normal in $H$. Further, if $L / Q^{\prime}$ is not cyclic then $H$ will contain at least two minimal normal subgroups isomorphic to $Q / M_{1}$ as $H$-modules, a contradiction. Thus $Q / Q^{\prime}=\left(X / Q^{\prime}\right) \times\left(Y / Q^{\prime}\right)$, wher $X, Y$ are normal in $H$ and we may assume $\left|X / Q^{\prime}\right|=q$. But then it follows from Taunt [14, Lemma 6.1 and Corollary 6.2] and the fact that $X / Q^{\prime}$ is not isomorphic to the chief factors of $H$ contained in $Q^{\prime}$ that we can find $S \leq X$ with $S \cap L=1$. Let $U_{Q}=U_{1} \oplus \cdots \oplus U_{t}$, where the $U_{i}$ are the homogeneous components. If $q$ divides $p-1$ then we have that $U_{Q^{\prime}}$ is not homogeneous since $Q^{\prime}$ is not central in $H$ and so we may suppose in this case that $U_{1 Q^{\prime}}$ is not isomorphic to $U_{2 Q^{\prime}}$. If $W$ is an irreducible constituent of $U_{Q}$, the Mackey subgroup theorem gives $W_{S}$ contains a trivial irreducible. Let $U_{1 S}=T_{1} \oplus Z_{1}, U_{2 S}=T_{2} \oplus Z_{2}$, where $T_{1}, T_{2}$ are trivial irreducible modules. Let $D$ be a diagonal subspace of $T_{1} \oplus T_{2}$ and set $V=D \oplus Z_{1} \oplus Z_{2} \oplus U_{3} \oplus \cdots \oplus U_{t}$. We now have $V$ is an $S$-submodule of $U$ 
with $U / V$ trivial. Clearly $Q^{\prime} \leq S^{H}$ and $V$ is not a $Q^{\prime}$-submodule of $U$. This completes the proof of the lemma.

LEMMA 7. Let $G$ be a group with $F(G)=O_{p}(G)$ nonabelian. Set $P=$ $O_{p}(G)$ and suppose that for some chief factor $A / B$ of $G$ with $\zeta(P) \leq B, A \leq$ $P$, we have $G / C_{G}(A / B)$ nonabelian. Then there is a subgroup $S$ of $O_{p}(G)$ with $S$ not normal in $S^{G}$.

Proof. Suppose the result is not true and choose $G$ to be a minimal counterexample. It is easy to see that $G$ must be monolithic. Set $H / P=$ $O_{p^{\prime}}(G / P)$ and let $Q$ be a Hall $p^{\prime}$-subgroup of $H$. Suppose that $C_{Q}(P / \zeta(P))$ $=D \neq 1$. Then we must have $[\zeta(P), D] \neq 1$ (since $[P, D] \neq 1$ ). But $\sigma(G) \leq P^{\prime}$ and hence $[\sigma(G), D]=1$. Since $\sigma(G) \leq \zeta(P)$, Lemma 1 gives a contradiction. Thus $D=1$.

Choose $M / P$ minimal such that $M / P$ is nonabelian and subnormal in $G / P$ and every subnormal subgroup of $M / P$ is abelian. Since $O_{p}(M / P)=1$ and $C_{M / P}(P / \zeta(P))=1$, we must have some chief factor $U / V$ of $M$ with $\zeta(P) \leq V, U \leq P$, with $M / C_{M}(U / V)$ nonabelian, a contradiction unless $M=G$. Thus we have $G / P$ has all subnormal subgroups abelian. If $G / P$ does not have a unique maximal normal subgroup then it will be nilpotent (since it is then the product of nilpotent normal subgroups). If $G / P$ is not a $p^{\prime}$-group it cannot be nilpotent and hence must have a unique maximal normal subgroup (which is then an abelian $p^{\prime}$-group) whose quotient has order $p$. In either case $(G / P)^{\prime}=X_{0} / P$ is an abelian $p^{\prime}$-group. Let $X$ be a Hall $p^{\prime}$-subgroup of $X_{0}$.

Let

$$
P / \Phi(P)=\left(U_{1} / \Phi(P)\right) \times \cdots \times\left(U_{t} / \Phi(P)\right),
$$

where the $U_{i} / \Phi(P)$ are indecomposable as $G$-modules. If $G / P$ is a $p^{\prime}$-group then all the $U_{i} / \Phi(P)$ are irreducible. If $G / P$ is not a $p^{\prime}$-group then $G / P$ is $p$-nilpotent and so all the composition factors of $U_{i} / \Phi(P)$ are isomorphic by Lemma 3. If $U_{i} / V$ is a nontrivial irreducible then it is an easy deduction from Blichfeldt's Theorem and the Mackey Subgroup Theorem that $U_{i} / V$ is projective and hence $U_{i} / \Phi(P)$ is irreducible.

Suppose that $P / R$ is a noncentral chief factor of $G$ with $G / C_{G}(P / R)$ abelian. Since $P / R$ is the $\mathscr{S}_{p^{\prime}} \mathscr{S}_{p}$ residual of $G / R, P / R$ is a complemented chief factor of $G$. Let $H$ be a complement for $P / R$ in $G$. Note that $R$ is the Fitting subgroup of $H$ and that $R$ contains a chief factor $A / B$ for $H$ with $H / C_{H}(A / B)$ nonabelian. The minimality of $G$ also gives that if $C / D$ is a chief factor of $H$ with $\zeta(R) \leq D, C \leq R, H / C_{H}(C / D)$ is abelian. If $R$ is nonabelian we get $\left[R^{\prime}, X\right]=1$. We have that $X R / R$ is a normal 
$p^{\prime}$-subgroup of $G / R$ and Lemma 1 then gives us that $\zeta(R)=U \times V$, with $[U, X]=1$ and $[V, X]=V$ and we have $U \neq 1, V \neq 1$. Since both $U$ and $V$ are normal in $G$ we have a contradiction. Thus $R$ must be abelian and if $A / B$ is any chief factor of $G$ with $A \leq R$ we cannot have $X \leq C_{G}(A / B)$ and so we must have $G / C_{G}(A / B)$ nonabelian. Again from the minimality of $G$ we see that $P / \sigma(G)$ must be abelian and hence $P$ has class 2.

Now suppose that no noncentral chief factor $P / R$ has $G / C_{G}(P / R)$ abelian. It is then clear that $\sigma(G)=P^{\prime}$. Thus $P$ must have class 2 and $\Phi(P) \leq \zeta(P)$.

We may suppose that $G / C_{G}\left(U_{1} / \Phi(P)\right)$ is nonabelian and $\zeta(P) \leq U_{2} \cdots U_{t}$. If $U_{1}$ is nonabelian and $x$ is an element of $U_{1}$ not in $\Phi(P)$ then $\langle x\rangle^{G} \Phi(P)$ $=U_{1}$ and so $\langle x\rangle^{G}$ is nonabelian. We can then choose $x$ so that $\langle x\rangle$ is not normal in $U_{1}$ and hence not normal in $\langle x\rangle^{G}$, a contradiction. Thus we have that $U_{1}$ is abelian and also that $t>1$. Moreover since $\left[U_{1}, X\right] \neq 1$ we have by Lemma 1 and the fact that $G$ is monolithic that $\left[U_{1}, X\right]=U_{1}$. We may also suppose that $\left[U_{1}, U_{2}\right] \neq 1$ since $U_{1}$ is not central in $P$.

Suppose that $U_{2} / \Phi(P)$ is not isomorphic to $U_{1} / \Phi(P)$ as $G$ module. We can then find an element $x$ in $U_{1} U_{2}$ but not in $U_{1}$ or $U_{2}$ with $\langle x\rangle$ not normal in $U_{1} U_{2}$ and $U_{1} U_{2}=\langle x\rangle^{G} \Phi(P)$ and hence $\langle x\rangle$ not normal in $\langle x\rangle^{G}$. Thus we must have $U_{1} / \Phi(P)$ and $U_{2} / \Phi(P)$ isomorphic as $G$ modules. Note that we may deduce as above that $U_{2}$ is abelian. Now consider $\Phi(P)$. Since it is central in $P$, we may regard it as a $G / P$ module. Also, all the chief factors of $G$ contained in $\Phi(P)$ are projective as $G / P$ modules and hence $\sigma(\Phi(P))$ is completely reducible as $G$ module. But $G$ is monolithic and so $\sigma(\Phi(P))$ is irreducible and it follows that $\Phi(P)$ is an indecomposable homocyclic subgroup. If $U_{i}$ is not indecomposable homocyclic, then $\sigma\left(U_{i}\right)>$ $P^{\prime}$ and $\sigma\left(U_{i}\right) \cap P^{\prime}$ is irreducible. Set $V_{i}=U_{i}$ if $U_{i}$ is an indecomposable homocyclic subgroup and $V_{i}=\sigma\left(U_{i}\right)$ otherwise. By relabelling if necessary, we may suppose that the exponent of $V_{1}$ is at least the exponent of $V_{2}$. If $P_{0}=V_{1} V_{2}$, then $V_{1}, V_{2}$ are abelian normal subgroups of $G$ and $P_{0}$ is nonabelian. Set $W_{i}=V_{i} \cap \Phi(P)$, then $X_{i}=V_{i} / W_{i}$ is a chief factor of $G$ and $\Phi\left(P_{0}\right)=W_{1} W_{2}$. Regarding $X_{1}$ as a $G F(p) G$-module we have $E=$ End $_{G F(p) G}\left(X_{1}\right)$ is a field and since $G / C_{G}\left(X_{1}\right)$ is nonabelian and of odd order, we have $\operatorname{dim}_{E}\left(X_{1}\right) \geq 3$ (using for example Curtis and Reiner [1, Theorem 53.17]). We have $P_{0} / \Phi\left(P_{0}\right)$ isomorphic to $X_{1} \oplus X_{2}$ as $G F(p) G$-module and then by Curtis and Reiner [1, Theorem 61.16] $P_{0} / \Phi\left(P_{0}\right)$ is generated (as module) by a single element. Thus we can find $u \in V_{1}, v \in V_{2}$ such that $\langle u v\rangle^{G}=P_{0}$. Then $\langle u v\rangle$ is normal in $P_{0}$ by hypothesis and so, setting $C_{0}=C_{P 0}(\langle u v\rangle), P_{0} / C_{0}$ is a nontrivial cyclic group. Moreover $\Phi\left(P_{0}\right)$ is 
central in $P_{0}$ giving $\left|P_{0} / C_{0}\right|=p$. If $\left[V_{1}, v\right]=1$ then $\left[V_{1}, v^{g}\right]=1$ for all $g \in G$, giving $\left[V_{1}, V_{2}\right]=1$, a contradiction. Thus, setting $C=C_{V 1}(v)$, we have $C \neq V_{1}$. On the other hand $C=C_{V 1}(u v)=C_{0} \cap V_{1}$, so that $\left|V_{1} / C\right|=p$. Since there is a one to one correspondence between the subgroup $Y / \Phi\left(P_{0}\right)=\left\{y v \Phi\left(P_{0}\right): y \in V_{1}, y v \Phi\left(P_{0}\right)\right.$ generates an irreducible submodule of $\left.P_{0} / \Phi\left(P_{0}\right)\right\}$ and $\operatorname{Hom}_{G F(p) G}\left(X_{1}, X_{2}\right)$, we have $\left|Y / \Phi\left(P_{0}\right)\right|=|E|$. Thus we cannot have $Y \supseteq C$. Choose $x$ in $V_{1} \backslash C$. Since $V_{1}$ is homocyclic and $C / \Phi\left(P_{0}\right)$ is not cyclic, we can choose a 2-generator homocyclic subgroup $W$ of $C$ with $W$ not contained in $\Phi\left(P_{0}\right)$ and not contained in $Y$. We can then choose $u, w \in W$ so that $W_{0}=\langle u v, w\rangle$ is a 2-generator homocyclic group of the same exponent as $W$. Since $W_{0}$ is homocyclic, we can choose $y \in W_{0}$ with $y=u^{r} w^{s} v$ and $\langle y\rangle \cap\langle[v, x]\rangle=1$. Then we have, setting $z=u^{r} w^{s}, y^{x}=y^{n}=z^{n} v^{n}$ for some integer $n$. However we also have $y^{x}=z^{x} v^{x}=z v^{x}$ and so $y^{n-1}=[v, x]$, a contradiction.

This completes the proof of the lemma.

\section{Proof of the theorem}

For the rest of this section $G$ denotes a fixed monolithic group of odd order in $\mathscr{B}_{2}$. If $G$ is nilpotent and hence a $p$-group that it follows from Heineken [5, Theorem 2] and Mahdavianary [9, Theorem B] that $G$ has class 3. Hence we suppose that $G$ is non-nilpotent.

We set $F(G)=P$ and suppose next that $P$ is nonabelian. Suppose further that $G / P$ is nonabelian. Set $M / P=(G / P)^{\prime} \cap O_{p^{\prime}}(G / P)$ then clearly $M \neq P$. If $[A, M] \leq B$ for every chief factor $A / B$ of $G$ with $\zeta(P) \leq B$ and $A \leq P$ then $\left[\zeta(P) \cap P^{\prime}, M\right]=1$. Since we cannot have $[A, M] \leq B$ for every chief factor of $G$ with $A \leq P$, we cannot have $[\zeta(P), M]=1$. Lemma 1 then gives $\zeta(P)=C \times D$ with $[C, M]=1,[D, M]=D$ and both $C$ and $D$ nontrivial and normal in $G$. This gives a contradiction since $G$ is monolithic. Thus $G$ satisfies the hypotheses of Lemma 7 and the conclusion of Lemma 7 tells us that $G$ cannot be in $\mathscr{B}_{2}$.

It follows that if $P$ is nonabelian then $G / P$ is abelian of order prime to $p$, establishing (ii). Thus we suppose that $P$ is abelian and we suppose also that $G$ has been chosen minimal such that (iii) is not satisfied.

If $S / R$ is a chief factor of $G$ with $S \leq P$ and $C_{G}(S / R)>P$ then we set $K / P=C_{G}(S / R) P \cap F(G / P)$. Then $S=C_{S}(K) \times[P, K]$ (by Lemma 1) and since $G$ is monolithic we must have $C_{S}(K)=1$ and then $K$ acts fixed point freely on $S / R$, a contradiction. Thus $C_{G}(S / R)=P$ and if $G / P$ has nilpotent length precisely $n$ then $P$ is the $\mathscr{N}^{n}$ residual of $G$ and so has a complement $H$ in $G$ by Huppert [7, Satz VI.7.15]. If $P$ is not minimal 
normal then the minimality of $G$ gives us that $\sigma(G) H$ has the structure given by (iii) and hence so does $H$. Thus we may assume that $P$ is minimal normal in $G$ and has a complement $H$ in $G$.

Let $N$ be a normal elementary abelian $q$-subgroup of $H, q$ a prime. Let $P_{N}=U_{1} \oplus \cdots \oplus U_{t}$, where the $U_{i}$ are the homogeneous components of $P_{N}$ and let $C_{i}=C_{N}\left(U_{i}\right)$; note that $\left|N / C_{i}\right|=q$. Suppose moreover that $q$ does not divide $p-1, N$ is the normal closure of a single element and that $|N| \geq q^{2}$; note that then $t>1$ and $\left|U_{i}\right|>p$. Let $D$ be any subgroup of $U_{1}$ of order $p$; note that $D$ is not normalised by $N$, but is normalised by $C_{1}$ and that $C_{1}^{H}=N$. Then, setting $E=\left(D \oplus U_{2} \oplus \cdots \oplus U_{t}\right) C_{1}$, we get $E$ subnormal in $G$ but not normal in its normal closure $P N$. Thus if $q$ does not divide $p-1$ then $N$ is cyclic. In fact more is true for if $q$ is a prime not dividing $p-1$ then $O_{q}(H)$ is cyclic. To see this we first observe that by Lemma $2 \sigma(H) \cap O_{q}(H)$ is generated by a single element as normal subgroup and so is cyclic. If $O_{q}(H)$ is not itself cyclic then suppose it contains a noncyclic elementary abelian normal subgroup, $A$ say. If $a \in A \backslash \sigma(H)$ then $\langle a\rangle^{H}$ is not cyclic, a contradiction. In particular, $\zeta\left(O_{q}(H)\right)$ is cyclic and so if $U$ is an irreducible constituent of $P_{O_{q}(H)}$ then $U$ is faithful. We then have $U O_{q}(H)$ in $\mathscr{B}_{2}$ and $O_{q}(H)$ contains a noncyclic elementary abelian subgroup by Huppert [7, Satz III.12.3] and as above we get a contradiction. Thus $O_{q}(H)$ is cyclic.

Now suppose that $q$ divides $p-1$ and that $O_{q}(H)$ is abelian. Then $O_{q}(H)$ contains a chief factor of $H$ of order at least $q^{3}$ if and only if $\sigma\left(O_{q}(H)\right)$ contains such a chief factor and if $\sigma\left(O_{q}(H)\right)$ contains such a chief factor there is a normal subgroup $N$ of $H$ contained in $\sigma\left(O_{q}(H)\right)$ with a unique maximal $H$-invariant subgroup $M$ and with $|N / M| \geq q^{3}$. If $W$ is a homogeneous component of $P_{N}$ and $C=C_{N}(W)$ then $C^{H}=N$ and Lemma 5 gives a contradiction. Thus if $A / B$ is a chief factor of $H$ with $A \leq O_{q}(H)$ then $|A / B| \leq q^{2}$.

Finally, suppose that $O_{q}(H)$ is nonabelian and suppose also that $O_{q}(H)$ contains a noncentral chief factor. Set

$$
M /\left(O_{q}(H) \times O_{q^{\prime}}(H)\right)=O_{q^{\prime}}\left(H / O_{q}(H) \times O_{q^{\prime}}(H)\right),
$$

then $N=O_{p^{\prime} p}(M)$. Then $N$ is $p$-nilpotent, $O_{q}(N)=O_{q}(H)$ and $O_{q}(N)$ contains noncentral chief factors of $N$. Also, if $W$ is an irreducible constituent of $P_{N}$ and $C=C_{N}(W)$ then $O_{q}(N / C)$ is nonabelian and contains noncentral chief factors of $N / C$. Lemma 6 (with $W$ and $N / C$ for $U$ and $H$ respectively) then tells us that $W(N / C)$ cannot be in $\mathscr{B}_{2}$, a contradiction. 


\section{References}

[1] C. W. Curtis and I. Reiner, Representation Theory of Finite Groups and Associative Algebras, (Pure and Applied Mathematics 11, Interscience Publishers, New York, London, 1962).

[2] C. Casolo, 'Gruppi finite risolubili in cui tutti i sottogrupi subnormali hanno difetto al pui 2', Rend. Sem. Mat. Univ. Padova 71 (1984), 257-271.

[3] W. Gaschütz, 'Gruppen in denen das Normalteilersein transitiv ist', J. Reine Angew. Math. 198 (1957), 87-92.

[4] _ , 'Endliche Gruppen mit treuen absolut-irreduziblen Darstellungen', Math. Nachr. 12 (1954), 253-255.

[5] H. Heineken, 'A class of three Engel groups', J. Algebra 17 (1971), 341-345.

[6] G. Higman, 'Complementation of abelian normal subgroups', Publ. Math. Debrecen 4 (1955-56), 455-458.

[7] B. Huppert, Endliche Gruppen I, (Die Grundlehren der Mathematischen Wissenschaften, Bd 134, Springer-Verlag, Berlin, Heidelberg, New York, 1967).

[8] B. Huppert and N. Blackburn, Finite Groups II, (Die Grundlehren der Mathematischen Wissenschaften, Bd 242, Springer-Verlag, Berlin, Heidelberg, New York, 1982).

[9] S. K. Mahdavianary, 'A special class of three Engel groups', Arch. Math. 40 (1983), 193-199.

[10] D. J. McCaughan and S. Stonehewer, 'Finite soluble groups whose subnormal subgroups have defect at most two', Arch. Math. 35 (1980), 56-60.

[11] T. A. Peng, 'Finite groups with pronormal subgroups', Proc. Amer. Math. Soc. 20 (1969), 232-234.

[12] D. J. S. Robinson, 'A note on finite groups in which normality is transitive', Proc. Amer. Math. Soc. 19 (1968), 933-937.

[13] _ A Course in the Theory of Groups, (Graduate Texts in Mathematics 80, SpringerVerlag, New York, Heidelberg, Berlin, 1982).

[14] D. R. Taunt, 'On A-groups', Proc. Cambridge Phil. Soc. 45 (1949), 24-42.

[15] G. Zacher, 'Caratterizzazione dei $t$-gruppi finiti risolubili', Ricerche Mat. 1 (1952), 287294.

\section{Department of Mathematics}

A.N.U.

GPO Box 4

Canberra, ACT 2601

Australia 